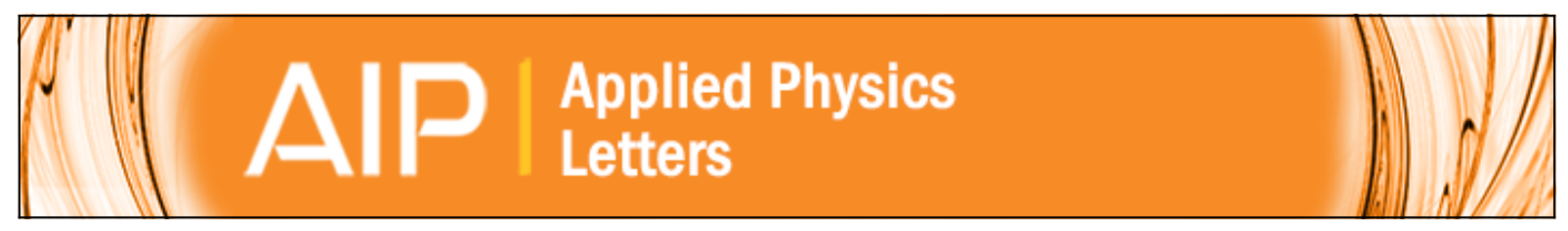

\title{
Production and characterization of a fully ionized He plasma channel
}

E. W. Gaul, S. P. Le Blanc, A. R. Rundquist, R. Zgadzaj, H. Langhoff, and M. C. Downer

Citation: Applied Physics Letters 77, 4112 (2000); doi: 10.1063/1.1329323

View online: http://dx.doi.org/10.1063/1.1329323

View Table of Contents: http://scitation.aip.org/content/aip/journal/apl/77/25?ver=pdfcov

Published by the AIP Publishing

\section{Articles you may be interested in}

Note: Characterization of the plasma parameters of a capillary discharge-produced plasma channel waveguide to guide an intense laser pulse

Rev. Sci. Instrum. 81, 046109 (2010); 10.1063/1.3397321

Measurement of ultrafast dynamics in the interaction of intense laser pulses with gases, clusters, and plasma waveguidesa)

Phys. Plasmas 12, 056712 (2005); 10.1063/1.1885005

Efficient generation of extended plasma waveguides with the axicon ignitor-heater scheme

Phys. Plasmas 11, L21 (2004); 10.1063/1.1695354

Modified electron cyclotron resonance source design for 3 He accelerator mass spectroscopy (abstract)

Rev. Sci. Instrum. 73, 887 (2002); 10.1063/1.1432461

Homogeneity characterization of a large microwave plasma

J. Vac. Sci. Technol. A 15, 668 (1997); 10.1116/1.580703

\section{Model PS-100}

Tabletop Cryogenic Probe Station 


\title{
Production and characterization of a fully ionized He plasma channel
}

\author{
E. W. Gaul, ${ }^{\text {a) }}$ S. P. Le Blanc, A. R. Rundquist, R. Zgadzaj, H. Langhoff, ${ }^{\text {b) }}$ \\ and M. C. Downer \\ University of Texas at Austin, Department of Physics, Austin, Texas 78712
}

(Received 12 June 2000; accepted for publication 2 October 2000)

\begin{abstract}
We report guiding of intense $\left(I=1.3 \pm 0.7 \times 10^{17} \mathrm{~W} / \mathrm{cm}^{2}\right) 80$ fs laser pulses with negligible spectral distortion through 1.5 -cm-long preformed helium plasma channels. Channels were formed by axicon-focused laser pulses of either $0.3 \mathrm{~J}$ energy, 100 ps duration, after preionizing a 200-700 Torr backfill of He gas to $n_{e} \sim 10^{16} \mathrm{~cm}^{-3}$ with a pulsed electrical discharge; or 0.6-1.1 J energy, $400 \mathrm{ps}$ duration, which required neither preionization nor intentional impurities for seeding. Transverse interferometry showed that $\mathrm{He}$ was fully ionized on the channel axis in both cases. Identical femtosecond pulses suffered substantial ionization-induced blueshifts after propagating through Ar and $\mathrm{Ne}$ channels of similar dimensions. (C) 2000 American Institute of Physics.
\end{abstract}

[S0003-6951(00)04648-9]

Plasma waveguides capable of guiding intense femtosecond (fs) laser pulses over multiple Rayleigh lengths without optical distortion are essential to developing practical laserdriven plasma accelerators and coherent short-wavelength light sources. ${ }^{1}$ Although sufficiently intense pulses can selfguide by relativistic self-focusing, ${ }^{2-4}$ preformed plasma channels $^{5-7}$ offer superior control over the mode quality of the guided pulse. ${ }^{8}$ Milchberg and co-workers ${ }^{6-8}$ have developed a method for producing $2 \mathrm{~cm}$ long, $10-50 \mu \mathrm{m}$ radius channels of excellent optical quality by ionizing and heating heavy noble gases or $\mathrm{N}_{2}$ with axicon-focused $0.3 \mathrm{~J}, 100 \mathrm{ps}$ $\mathrm{Nd}: \mathrm{Y}_{3} \mathrm{Al}_{5} \mathrm{O}_{12}$ (Nd:YAG) laser pulses to induce a radially expanding shock. However, these pulses generally leave the channel only partially ionized, and its entrance/exit regions nearly un-ionized. Consequently, ionization-induced phase modulation can distort intense fs pulses propagating through the channel and end regions. Solutions to this problem so far have been either expensive (e.g., generating, in addition to channel forming and guided pulses, a third intense pulse to completely preionize the channel), incomplete (e.g., finding conditions for which most channel ions are stripped to noble gas cores, thus leaving an ionization-free intensity "window"), or limited in length scalability (truncating end regions by using a gas jet). ${ }^{9,10}$ In this letter, we demonstrate distortion-free propagation of $0.3 \mathrm{TW}$ laser pulses through $1.5 \mathrm{~cm}$ long, fully ionized He plasma channels and their unionized end regions. Past channel-forming pulses lacked the peak intensity to field-ionize enough $\mathrm{He}$ atoms to seed avalanche ionization (AI) - the main breakdown mechanism-by the same pulse. We have overcome this problem by either: (1) striking a $2 \mathrm{~cm}$ long preplasma $\left(n_{e}\right.$ $\sim 10^{16} \mathrm{~cm}^{-3}$ ) in a He backfill with an inexpensive pulsed electrical discharge, thus seeding AI by our synchronized 100 ps, 0.3 J Nd:YAG channel-forming pulse; or (2) lengthening the Nd:YAG pulse to $400 \mathrm{ps}$ and amplifying it to $\simeq 0.6 \mathrm{~J}$ so that $\mathrm{AI}$ and inverse bremsstrahlung (IB) heating

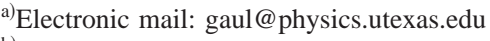

${ }^{b)}$ Permanent address: Physikalisches Institut der Universität Würzburg, 97074 Würzburg, Germany. have sufficient time to build up even from field ionized trace contaminants $\left(n_{e}^{\text {con }} \ll 10^{16} \mathrm{~cm}^{-3}\right)$ without a preplasma. In addition the high field-ionization threshold $\left(I_{P}=24.6 \mathrm{eV}\right)$ of He suppresses ionization by the guided pulse in the end regions. Thus we can form a distortion-free channel in a backfill, which is more length scalable and uniform in density than a gas jet. Ionization in $\mathrm{H}_{2}$ occurs $\sim 3.5 \times$ further from the focus than in $\mathrm{He}$, giving $\mathrm{He}$ the advantage of a shorter coupling region.

Figure 1 shows a schematic of our experiment. The 100 ps, $0.3 \mathrm{~J}$ Nd:YAG laser pulse (P1) from a self-filtering regenerative amplifier ${ }^{11}$ is line focused by an axicon lens to generate a plasma channel. An $\sim 30 \mathrm{~mJ}, 80 \mathrm{fs}$ Ti:S laser pulse (P2) is focused with an $f / 8$ off-axis parabolic mirror, reflected from a dichroic mirror into a $2 \mathrm{~mm}$ diameter axial hole in the axicon and coupled into the channel. Its transverse mode entering or exiting the channel is imaged onto CCD1 by L1 or into a spectrometer by L2. The channel profile is probed transversely by a Mach-Zehnder interferometer using probe pulse (P3), which is split from P1 and delayed $-3 \mathrm{~ns}<\Delta T 2<+1 \mathrm{~ns}$. The interferogram is imaged

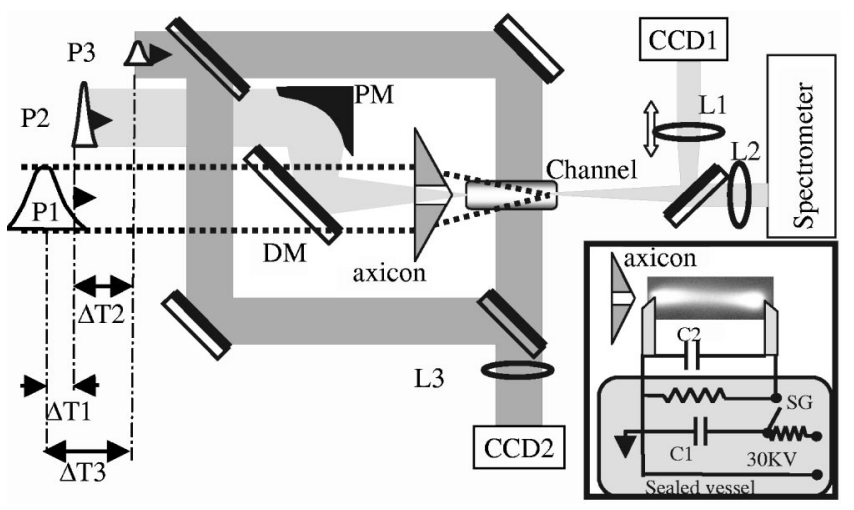

FIG. 1. Experimental layout. P1: Nd:YAG channel-forming pulse. P2: 80 fs, 0.4 TW Ti:S pulse coupled into channel by parabolic mirror PM and dichroic mirror DM through hole in axicon. P3: weak 80 fs pulse to probe $n_{e}(r)$ in channel interferometrically. CCD1, 2 record guided mode and interferograms imaged by lenses L1, L3, respectively. Inset: discharge circuit used to preionize $\mathrm{He}$; shaded area is pressurized to $2-3 \mathrm{~atm} \mathrm{~N}_{2}$. 
(a)
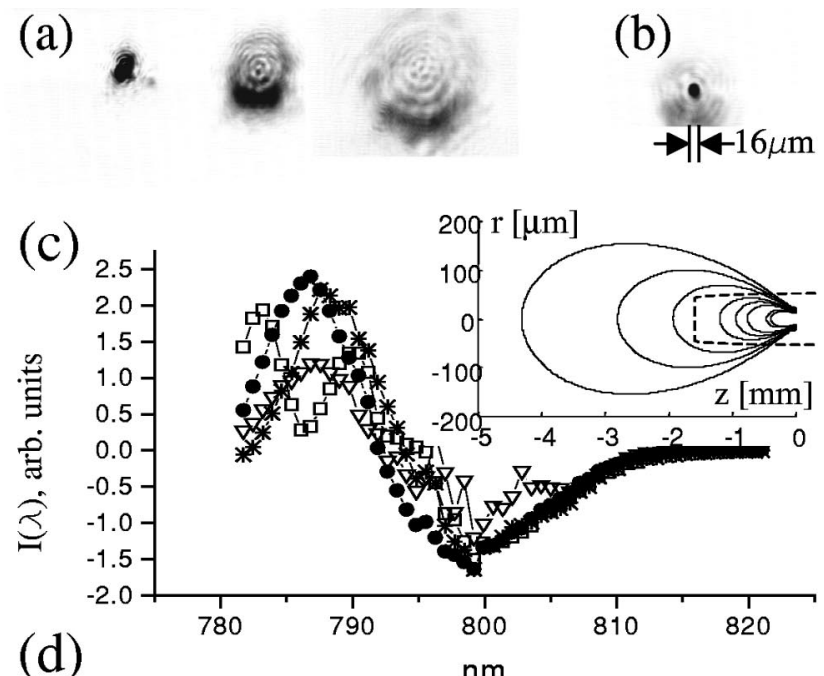

(d)

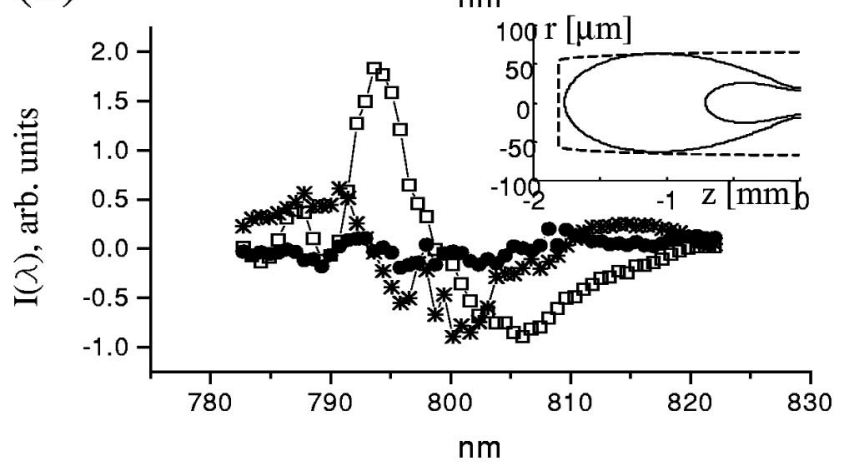

FIG. 2. Typical interferograms (a), (b) of He channels and corresponding $n_{e}(\mathbf{r})$ in $\mathrm{cm}^{-3}$ (c), (d) for $\Delta T 3=200 \mathrm{ps}$ and $\Delta T 3=6 \mathrm{~ns}$. Note the different scales on panel c vs d. Dotted curve in d: predicted guided mode in parabolic approximation $\left(w_{0}=14 \mu \mathrm{m}\right)$, dashed lines: $e^{-2}$ width of the measured mode $\left(w_{0}=8 \mu \mathrm{m}\right)$.

onto CCD2 by L3, evaluated by a Fourier-transform method $^{12}$ to extract the phase shift profile, then Abel transformed ${ }^{13}$ to yield an electron density profile $n_{e}(r)$. Jitter in the electronically adjusted delay $\Delta T 1$ between P1 and P2 is held to $\pm 200 \mathrm{ps}$ accuracy by controlling the Ti:S oscillator length with a piezoelectric transducer.

The inset shows the electrical discharge circuit used to preionize the He. The charge on a $0.5 \mathrm{nF}, \simeq 30 \mathrm{kV}$ storage capacitor $\mathrm{C} 1$ is held off by a spark gap (SG), which is enclosed in a sealed chamber that is pressurized with $2-3$ atm $\mathrm{N}_{2}$ and triggered by a synchronized $10 \mathrm{~mJ}, 8 \mathrm{~ns}$ laser pulse. When SG conducts, charge flows onto a second capacitor $\mathrm{C} 2$, thereby building up voltage across the $2 \mathrm{~cm}$ gap between two knife-edge electrodes that protrude into the He backfill near the axicon. The low inductance $(<100 \mathrm{nH})$ circuit builds up overvoltage quickly, triggering rapid $(\simeq 30 \mathrm{~ns}$ ) breakdown of the He and depositing $100 \mathrm{~mJ}$ into a plasma column (conductivity $\sigma \sim 0.1 \Omega^{-1}$ ) of initial radius $r_{i}$ $\simeq 0.1 \mathrm{~mm}$, which expands later to $r_{f} \sim 1 \mathrm{~mm}$, as seen in the time integrated luminescence image (Fig. 1, inset). From $\sigma$ and $r_{i}$ we estimate $n_{e} \simeq 10^{16} \mathrm{~cm}^{-3}$ and electron temperature $k T_{e} \simeq 1 \mathrm{eV}$, sufficient to absorb $>1 \%$ of the energy of P1 at $I=10^{13} \mathrm{~W} / \mathrm{cm}^{2}$ in $200-750$ Torr $\mathrm{He}$, which generates a plasma filament over $\sim 1.5 \mathrm{~cm}$ of the preionized region. The laser pulse formed no detectable filament without the discharge. Precise spatial $( \pm 20 \mu \mathrm{m})$ and temporal ( $\pm 5 \mathrm{~ns}$ )

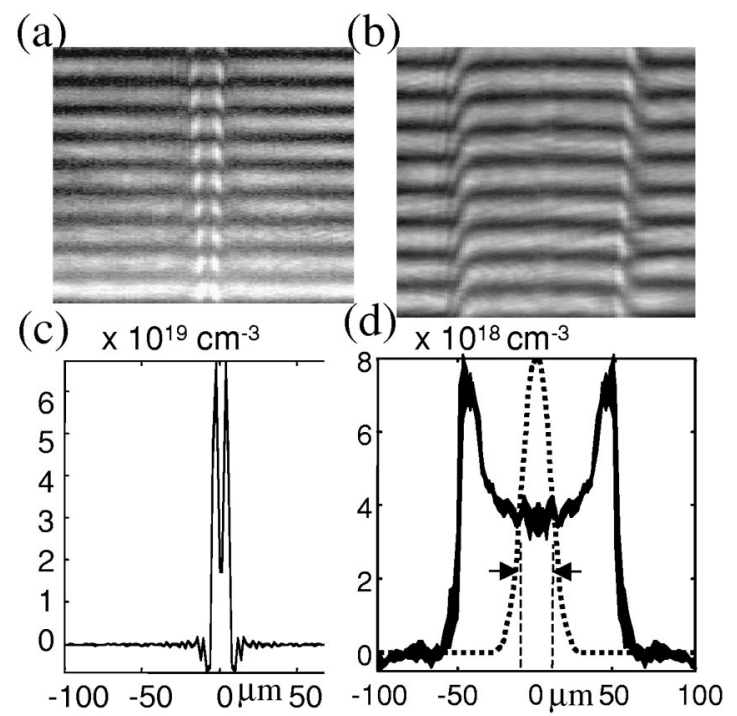

FIG. 3. Images of (a) expanding unguided transverse laser modes at positions $0-4 \mathrm{~mm}$ and (b) exiting He guided mode at $12 \mathrm{~mm}$ after the vacuum focus. Insets (c) and (d): difference spectra of Ti:S laser pulse after channel in $\operatorname{Ar}[(\mathrm{c})(--) 200$ Torr, $(-*-) 100$ Torr, $(-\nabla-) 50$ Torr $]$ and $[\mathrm{He}$ (d) (- - ) 750 Torr, $(-*-) 630$ Torr] backfill pressures. Squares: unchanneled pulses in 200 Torr Ar; 750 Torr He. Insets in (c), (d): isointensity contours corresponding to ionization levels of Ar, He, respectively, for Ti:S pulses focused to $I_{\text {guided }}=2 \times 10^{17} \mathrm{~W} / \mathrm{cm}^{2}$. Dashed curve: boundaries of plasma channel.

overlap of discharge with the laser pulse were found to be essential.

Longer heating pulses of equal peak intensity should produce equivalent plasma filaments from lower seed $n_{e} \cdot{ }^{14}$ To produce such a pulse we inserted a $3 \mathrm{~mm}$ solid fused silica etalon before the Nd:YAG regenerative amplifier to stretch the pulse to $400 \mathrm{ps}$ and added one amplifier stage to obtain up to $1.1 \mathrm{~J}$. We observed that $0.6-1.1 \mathrm{~J}$ pulses create plasma channels in He without any additional preseeding device. Only $n_{e} \sim 10^{10} \mathrm{~cm}^{-3}$ is required to seed efficient avalanche ionization of our interaction region by this laser pulse. Nevertheless, our 400 ps pulse cannot field ionize ${ }^{15}$ even this density in pure He. Since our chamber base pressure is $\sim 10$ mTorr, field ionization of low $I_{P}$ trace contaminants evidently supplies the seed electrons. For 100 ps pulses much higher partial pressure of low $I_{P}$ (but not fully ionizable) contaminants must be introduced. ${ }^{6,7}$

Figures 2(a) and 2(b) show interferograms, and Figs. 2(c), and 2(d) corresponding $n_{e}(r)$ profiles, of filaments formed in 750 Torr $\mathrm{He}$ at $\Delta T 3=200 \mathrm{ps}$ and $6 \mathrm{~ns}$ after the peak of a $400 \mathrm{ps}$ pulse. The $200 \mathrm{ps}$ profile shows a $10 \mu \mathrm{m}$ radius plasma column of average $n_{e}=6 \times 10^{19} \mathrm{~cm}^{-3}$, i.e., fully ionized $\mathrm{He}$, immediately following the Nd:YAG pulse. An electron shockwave then spreads out radially, creating the $n_{e}(r)$ profile in Fig. 2(d), which contains nearly the same integrated number $\int 2 \pi r n_{e}(r) d r$ of electrons per unit length as Fig. 2(c). When this profile is approximated by a parabola, the dashed curve in Fig. 2(d) shows the intensity profile of the lowest-order Gaussian mode $\left(e^{-2}\right.$ radius $\left.w_{0}=14 \mu \mathrm{m}\right)$ expected to be stably guided.

Figure 3(a) shows images of the transverse mode of P2 as the object plane of L1 was translated $0-4 \mathrm{~mm}$ from the focus of P2 with no channel present, and thus shows the expanding mode of a normal vacuum focus. The data in Fig. 
3(b) show the mode near the channel exit, at a location $\sim 12 \mathrm{~mm}$ from the focus, which is a typical exiting guided mode of radius $w_{0} \sim 8 \mu \mathrm{m}$ which we measure to contain $50 \%$ of the energy of the entering pulse, implying guided intensity $I_{\text {guided }}=2 \times 10^{17} \mathrm{~W} / \mathrm{cm}^{2}$. The discrepancy with the expected $14 \mu \mathrm{m}$ mode diameter (which implies $I_{\text {guided }}=0.6$ $\times 10^{17} \mathrm{~W} / \mathrm{cm}^{2}$ ) may originate from uncertainty in the Abeltransformed $n_{e}(r)$, but is not quantitatively understood. Thus we estimate $I_{\text {guided }}=1.3 \pm 0.7 \times 10^{17} \mathrm{~W} / \mathrm{cm}^{2}$.

In order to evaluate ionization-induced distortion, we compared the spectral blueshifts of intense $(I=2$ $\times 10^{17} \mathrm{~W} / \mathrm{cm}^{2}$ ) pulses exiting $\mathrm{Ar}, \mathrm{Ne}, \mathrm{N}_{2}$, and He channels of varying density. The results for $\mathrm{Ar}$ and $\mathrm{He}$ are shown by the differential power spectra $\Delta I(\lambda)=I_{\text {exit }}(\lambda)-I_{\text {in }}(\lambda)$ in Figs. $3(\mathrm{c})$ and 3(d), respectively. We compare He pressures four times larger than corresponding Ar pressures to keep the electron number approximately the same. When the incident pulses were focused into neutral gas without a channel, we observed substantial ionization-induced blueshifts for all gases, as shown by the open squares in Figs. 3(c) and 3(d). Intense guided pulses exiting $1.5 \mathrm{~cm}$ long (or 60 Rayleigh lengths for $w_{0}=8 \mu \mathrm{m}$ ) Ar channels were also strongly blueshifted throughout the investigated density range, as shown by the remaining data sets in Fig. 3(c). Very similar results were obtained for $\mathrm{Ne}$ and $\mathrm{N}_{2}$ channels. On the other hand, identical pulses exiting $\mathrm{He}$ channels showed significantly weaker blueshifts [stars, Fig. 3(d)] or, in some cases, no detectable blueshift whatsoever [filled circles, Fig. 3(d)].

The persistent blueshifts observed with $\operatorname{Ar}\left(\mathrm{Ne}, \mathrm{N}_{2}\right)$ channels can be attributed in part to ionization within the channel. Since the energy to strip the outer shell of $\mathrm{Ar}(\mathrm{Ne}$, $\mathrm{N}_{2}$ ) is much higher than that required to fully ionize $\mathrm{He}$, the Nd:YAG pulse evidently leaves that channel only partially ionized.

In addition, the focusing pulse ionizes neutral Ar while approaching the channel entrance. The inset of Fig. 3(c) shows that the isointensity contours (solid curves) corresponding to various field-ionization levels of Ar extend as much as 17 Rayleigh lengths into the neutral Ar preceding the plasma column (dashed curve) for $I_{\text {guided }}=2$ $\times 10^{17} \mathrm{~W} / \mathrm{cm}^{2}$. Ionizing propagation through this distance (mirrored at the exit) creates blueshifts approaching those of the unchanneled pulse. Both sources contribute to the observed blueshifts, although the present results do not distinguish their relative importance quantitatively.

For He channels, on the other hand, the full ionization evident from interferometry eliminates blueshifting within the channel. Equally important, the isointensity contours corresponding to field-ionization levels are much smaller than for Ar. In fact, as shown by the inset of Fig. 3(d), they fit approximately within the $50 \mu \mathrm{m}$ radius plasma column present at $\Delta T_{3}=6 \mathrm{~ns}$. Thus, with strategic adjustment of the focus of $\mathrm{P} 2$, ionization-induced distortions in the entrance region can be almost completely eliminated, as suggested by the result (filled circles) in Fig. 3(d). For the results obtained at $p_{\mathrm{He}}=630$ Torr $(-*-)$, although the interferometer still indicated full ionization in the middle of the channel, a shorter channel formed. However, the focus of P2 was not moved, and thus no longer coupled to a preionized position. The weak spectral distortion observed in this case can therefore be attributed to ionization in the end region. Similar distortions were observed as the focus of P2 was translated along the channel axis. At intensites $\geqslant 10^{18} \mathrm{~W} / \mathrm{cm}^{2}$, relativisitic plasma nonlinearities begin to distort the pulse. ${ }^{16}$

In summary, we have demonstrated that intense ultrashort pulses couple into, guide at $I=1.3 \pm 0.7 \times 10^{17} \mathrm{~W} / \mathrm{cm}^{2}$ and couple out of a fully ionized, $1.5 \mathrm{~cm}$ long plasma channel formed in a He backfill without spectral distortion. The channel-forming techniques used here are scalable to channel lengths of $\sim 10 \mathrm{~cm}$. This will enable studies of laser wakefield acceleration into and beyond the dephasing $\operatorname{limit}^{17}$ for $n_{e} \leqslant 10^{18} \mathrm{~cm}^{-3}$.

This research was supported by U.S. Department of Energy Grant No. DEFG03-96-ER-40954.

${ }^{1}$ P. Sprangle and B. Hafizi, Phys. Plasmas 6, 1683 (1999), and references therein

${ }^{2}$ K. Krushelnick, A. Ting, C. I. Moore, H. R. Burris, E. Esarey, P. Sprangle, and M. Baine, Phys. Rev. Lett. 78, 4047 (1997).

${ }^{3}$ R. Wagner, S.-Y. Chen, A. Maksimchuk, and D. Umstadter, Phys. Rev. Lett. 78, 3125 (1997).

${ }^{4}$ J. J. Fuchs, G. Malka, J. C. Adam, F. Amiranoff, S. D. Baton, N. Blanchot, A. Hron, G. Laval, J. L. Miquel, P. Mora, H. Ppin, and C. Rousseaux, Phys. Rev. Lett. 80, 1658 (1998).

${ }^{5}$ T. Hosokai, M. Kando, H. Dewa, H. Kotaki, S. Kondo, N. Hasegawa, K. Nakajima, and K. Horioka, Opt. Lett. 25, 10 (2000), and references therein.

${ }^{6}$ C. G. Durfee and H. M. Milchberg, Phys. Rev. Lett. 71, 2409 (1993).

${ }^{7}$ C. G. Durfee, J. Lynch, and H. M. Milchberg, Phys. Rev. E 51, 2368 (1995)

${ }^{8}$ T. R. Clark and H. M. Milchberg, Phys. Rev. Lett. 81, 357 (1998).

${ }^{9}$ S. P. Nikitin, I. Alexeev, J. Fan, and H. M. Milchberg, Phys. Rev. E 59, R3839 (1999).

${ }^{10}$ P. Volfbeyn, E. Esarey, and W. P. Leemans, Phys. Plasmas 6, 2269 (1999).

${ }^{11}$ P. G. Gobbi and G. C. Reali, Opt. Commun. 52, 195 (1984).

${ }^{12}$ M. Takeda, H. Ina, and S. Kobayashi, J. Opt. Soc. Am. 72, 156 (1980).

${ }^{13}$ G. Pretzler, Z. Naturforsch., A: Phys. Sci. 46, 639 (1991).

${ }^{14}$ N. E. Andreev, L. Y. Margolin, I. V. Pleshanov, and L. N. Pyatnitskii, Quantum Electron. 28, 910 (1998).

${ }^{15}$ M. V. Ammosov, N. B. Delone, and V. P. Kranov, Sov. Phys. JETP 64, 1191 (1986)

${ }^{16}$ P. Sprangle, B. Hafizi, and J. R. Peano, Phys. Rev. E 61, 4381 (2000).

${ }^{17}$ W. Leemans, C. W. Siders, E. Esarey, N. E. Andreev, G. Shvets, and W. B. Mori, IEEE Trans. Plasma Sci. 24, 331 (1996). 